\title{
Physical Frailty and Sarcopenia: Development of a Framework for Supporting Interventions Against Incident Mobility Disability
}

\author{
Matteo Cesari ${ }^{1,2}$ \\ ${ }^{1}$ Gérontopóle, Centre Hospitalier Universitaire de Toulouse, Toulouse, ${ }^{2}$ Université de Toulouse III Paul Sabatier, Toulouse, France
}

Corresponding Author:

Matteo Cesari, MD, PhD

Université de Toulouse III Pau

Sabatier, Faculté de Medicine,

37 Allées Jules Guesde, 31000

Toulouse, France

Tel: +33-5-6114-5628

Fax: +33-5-6114-5640

E-mail: macesari@gmail.com

Received: April 15, 2017

Revised: May 15, 2017

Accepted: May 17, 2017
In 2014, the Innovative Medicines Initiative funded a 48-million Euros project entitled "Sarcopenia and Physical fRailty IN older people: multi-componenT Treatment strategies” (SPRINTT). The SPRINTT consortium federates partners from academia, industry, and the private sector to address an important gap in the geriatric field: the lack of a standardized and clinically relevant definition of sarcopenia. In particular, the SPRINTT project aims to involve all major stakeholders in the framing of a skeletal muscle-specific risk profile that exposes the individual at risk of negative health-related outcomes (in particular, disability), is amenable to benefiting from preventive interventions, and meets the standards requested by regulatory agencies. During the first year of activity, the SPRINTT consortium has implemented a "Physical Frailty \& Sarcopenia" (PF\&S) condition that considers existing evidence and regularly exchanges information with the European Medicines Agency. This condition is currently the study objective of a 3-year randomized controlled trial in multiple countries across Europe. The trial aims to verify whether a multicomponent intervention (based on physical activity and nutritional counseling) can prevent the onset of mobility disabilities in community-dwelling older persons with PF\&S. The present article provides an overview of the SPRINTT background and rationale and explains the details of the PF\&S condition.

Key Words: Prevention, Elderly, Skeletal muscle, Mobility, Disability

\section{INTRODUCTION}

In 1968, an article entitled "Old and frail" appeared in British Medical Journal ${ }^{1)}$. At its very beginning, it stated, "one of the miracles of our time is the increase in the average length of life". A few lines later, it discussed how aging of the English and Welsh populations was generating new and worrisome scenarios for social and health care systems. At the end of the article, the authors admitted, "at present, the ills of old age are not adequately provided for." It is quite remarkable how that paper almost 50 years ago discusses the pressing issues that are still affecting care services. Besides showing that our healthcare systems have been struggling with the consequences of global aging for longer than what it is usually thought, the use of the word "frail" in the title is also noteworthy. In fact, 2001 is commonly identified as the "year of birth" of the so-called frailty condition. This association is mainly due to the appearance of the first operational definitions of frailty in the literature right during that year ${ }^{2,3)}$. In reality, as exemplified by the 1968 article, frailty had already been debated and considered in the geriatric field for many years. It can be said that frailty is a clinical condition deeply nested in the culture of geriatric medicine, a specialty finding its natural field of interest at the edge between normal and pathological aging ${ }^{4)}$.

\section{WHAT IS FRAILTY?}

There is unanimous agreement around the theoretical definition of frailty as provided by an international panel of experts in 2013 ${ }^{5}$. In this document, frailty was described as "a clinical state in which there is an increase in an individual's vulnerability for developing increased dependency and/or mortality when exposed to a stressor". Unfortunately, a huge controversy exists when this concept is translated from theory into instruments allowing its objective assessment. Over the years, multiple tests, questionnaires, and scales have been proposed and validated for capturing the frailty condition in older persons. On one hand, the high 
number of developed tools demonstrates the great (and growing) interest in the frailty condition. On the other hand, such abundance has affected the possibility of a single and straightforward discussion on the topic. In particular, although most of the available instruments are predictive of negative health-related outcomes, the agreement of their results remains quite modest ${ }^{6,77}$. In other words, every instrument can be considered legitimate (especially given the absence of a gold standard), but "talking multiple languages" at the same time is often misleading and potentially generates confusion. It is paradigmatic, for example, how often frailty describes the structure of a specific instrument instead of giving more consideration to its theoretical and biological foundations ${ }^{4)}$.

\section{WHAT IS SARCOPENIA?}

The term "sarcopenia" was originally proposed by Irwin Rosenberg. He stated that "no decline with age is as dramatic or potentially more significant than the decline in lean body mass" and "there may be no single feature of age-related decline more striking than the decline in lean body mass in affecting ambulation, mobility, energy intake, overall nutrient intake and status, independence and breathing. ${ }^{81}$ Interestingly, the ubiquitous presence of sarcopenia across living species makes this phenomenon of special interest for translational research on aging ${ }^{9,10}$. It has been proposed that sarcopenia might indeed represent a promising organ-specific pathophysiological background for defining a muscular condition of clinical relevance ${ }^{111}$. In this context, it is noteworthy that the recent allocation of a specific International Statistical Classification of Diseases and Related Health Problems (ICD10) code to sarcopenia ${ }^{12)}$ demonstrated how this condition has all the necessary characteristics for theoretically framing a nosological entity. However, the creation of an ICD-10 code for sarcopenia has surely solved part of the issue related to the implementation of the condition in the clinical setting. However, the code is still an orphan of a clear operational definition. In fact, regarding frailty, several measures and methodologies have been proposed in the literature for defining sarcopenia and no gold standard is unanimously accepted. It is indeed crucial to move ahead in the field and achieve agreement about how to measure sarcopenia in a standardized and validated way. This step is crucial for paving the way to the development and future use of interventions directed against the age-related skeletal muscle decline ${ }^{13)}$.

\section{OVERLAP BETWEEN FRAILTY AND SARCOPENIA}

Sarcopenia and frailty have always been studied in parallel despite a clear overlapping between them ${ }^{14}$. Sarcopenia has mostly been targeted by basic research scientists focused on skeletal muscle and aging. Differently, the concept of frailty has frequently been adopted in clinical settings ${ }^{15)}$ or for public health purposes ${ }^{16)}$.

The need exists for pharmacological and nonpharmacological interventions aimed at preventing skeletal muscle declines and functional losses. Indeed, several molecules and treatments are currently under development for this purpose (e.g., selective androgen receptor modulators, myostatin inhibitors). Nevertheless, actions in this direction are still hampered by the heterogeneous modalities that quantitatively and qualitatively measure the skeletal muscle modifications in a standardized and clinically relevant fashion. In particular, the current constructs of both frailty and sarcopenia are difficult to be accepted by regulatory agencies, which are used to deal with diseases of adulthood characterized by a clear, unidimensional, and straightforward pathophysiological mechanism. It is also noteworthy that the clinical relevance of frailty is strongly related to its multidimensional construct, whereas the age-related shrinking of the skeletal muscle is better characterized from a biological perspective but may not represent a priority in medical interventions ${ }^{17}$.

Discussions between researchers/clinicians and regulatory agencies seem stalled. However, it might be possible to consider frailty and sarcopenia as complementary and benefit from the positive aspects of each of the 2 constructs. In fact, a central core shared by the 2 conditions might support the development of a clinically relevant and organ-specific pathophysiological manifestation: skeletal muscle-related physical impairment. In this context, it cannot be underestimated the fact that several adverse outcomes of frailty are probably mediated by sarcopenia. This indicates that sarcopenia may represent the biological substrate for the development of physical frailty and/or the pathway through which the negative health outcomes of frailty ensue.

\section{THE "SPRINTT" PROJECT}

In 2014, the Innovative Medicines Initiative (IMI) funded the "Sarcopenia and Physical fRailty IN older people: multicomponenT Treatment strategies" (SPRINTT) project. The IMI is a European agency that functions as a mediator between the European Commission and the European Federation of Pharmaceutical Industries and Associates (EFPIA) to develop private-public research consortia for the generation of $a d$ hoc collaborations on innovative fields of research with special clinical relevance. The SPRINTT project was retained among several submitted applications and received an overall 48-million-Euro grant (50\% from the European Commission, $50 \%$ in-kind from the EFPIA). SPRINTT is specifically designed to overcome the existing barriers for an efficient public health intervention against frailty and support the implementation 
of strategies promoting successful aging across Europe. For this reason, the actions of the SPRINTT consortium involve all of the major stakeholders (i.e., academia, regulators, industry, and patients' representatives) and address several multifaceted objectives, including: (1) formulation of a clear operationalization of the presently vague concept of frailty, (2) identification of a target population with clear unmet medical needs, and (3) definition of an experimental setting as a template for regulatory purposes and pharmaceutical investigations in the field ${ }^{18)}$. The main aim is to arrive at the end of the project with a valid and clinically relevant definition for the assessment of the age-related skeletal muscle abnormality in agreement with the regulatory standards.

Thus, during its first year of activity, the SPRINTT project has proposed a novel operationalization of physical frailty recognizing sarcopenia as its central biological substrate: the physical frailty and sarcopenia (PF\&S) condition ${ }^{14)}$. For sure, the characterization of a definite biological basis (i.e., skeletal muscle decline and loss of mobility function) for frailty restricts this condition to the physical domain. However, this approach is necessary for opening new and dedicated venues for the possible development of interventions aimed at specifically slowing or reversing the skeletal muscle-related physical function decline. In this context, the SPRINTT consortium has reviewed available literature, interacted with major stakeholders, and exchanged information with regulatory agencies (in particular, the European Medicines Agency).

The identification of the population of interest for SPRINTT will rely on the 3 key elements of the PF\&S operationalization: (1) target organ deterioration (i.e., skeletal muscle assessment), (2) clinical manifestation of physical frailty (i.e., reduced physical performance), and (3) preserved functional status (i.e., absence of mobility disability $)^{19}$. The rationale underlying the operational construct of the PF\&S components is detailed in the following section entitled "The rationale for defining the PF\&S condition."

A randomized controlled clinical trial (RCT) was started in SPRINTT in February 2016. The RCT aimed to verify the construct validity of the hypothesized PF\&S condition and verify the possibility of using it as a target for preventive strategies against disability. The methodology and design of the RCT were recently published elsewhere ${ }^{20)}$. Briefly, the RCT recruits 1,500 participants aged $\geq 70$ years distributed across 11 European countries. The target population is composed of community-dwelling older persons with PF\&S (i.e., nondisabled but exposed to increased vulnerability to stressors due to their skeletal muscle decline). SPRINTT participants were randomized to a multicomponent intervention (based on a structured moderate-intensity physical activity program plus nutritional counseling; $n=750$ ) or a health education program (consisting of workshops on topics relevant to older adults; $n=750$ ) and followed up for 2-3 years. The primary outcome of the trial is the incidence of mobility disability, but several other major negative health-related events (including mortality, hospitalizations, institutionalizations, and healthcare expenses) are also monitored as secondary interests.

Besides the key elements composing this definition, the eligibility criteria adopted in the SPRINTT trial are aimed at: (1) excluding persons with specific clinical conditions that may render the intervention unsafe (i.e., severe organ system diseases and unstable health status) and (2) avoiding the inclusion of individuals whose adherence to the protocol might be low due to clinical (e.g., cognitive impairment and chronic dialysis) and/or nonclinical reasons (e.g., plans to relocate outside the study area). It is important to mention that the SPRINTT trial participants will be included because of frailty and not because of specific diseases. In other words, the eligibility is judged based on a functional assessment rather than the diagnosis of nosological conditions. This means that as soon as a person presents the condition of interest (i.e., PF\&S) and the potentialities for benefiting from a preventive intervention against incident disability, he/she is likely to be eligible for SPRINTT. Besides rendering the study sample very representative of a "real life" older adult population, this approach is intended to prioritize the relevance of function over that of nosological conditions ${ }^{211}$.

\section{RATIONALE FOR DEFINING PF\&S}

It is common sense that the choice of an instrument for measuring a certain risk condition should be made according to the actions that will be implemented based on the positivity or negativity of its results. In other words, the choice of the instrument should consider the available evidence but will also be driven by the needs, objectives, and resources of the evaluation. In the fields of frailty and sarcopenia, where no gold standard exists for operationally capturing the phenomena, the assessment tool to be chosen is strongly related to "what will happen next"

In the context of the SPRINTT project, the consortium needed to define a condition of risk predisposing the older person at risk of incident disability while being strongly characterized by a muscular pattern. Therefore, the group explored the different possibilities offered by the multitude of instruments/tools to triangle: (1) a marker of skeletal muscle decline, (2) a measure of physical impairment, and (3) a criterion for excluding the presence of established disability.

\section{Skeletal Muscle Decline}

Up until a few years ago, the objective assessment of sarcopenia was largely modeled around expert consensus opinions $^{22-25)}$. Despite sharing some commonalities (e.g., pro- 
posing the simultaneous assessment of a quantitative and qualitative marker of skeletal muscle), these recommendations were affected by the lack of strong data supporting them; they were indeed consensus (thus somehow arbitrary) recommendations. Not surprisingly, when the reports by the Foundation for the National Institutes of Health-Sarcopenia Project $(\mathrm{FNIH})$ initiative $^{26)}$ were released, they indeed generated a revolution in the field. In fact, the FNIH recommendations were based on the results of ad hoc analyses conducted in multiple cohort databases of older persons. The adopted classification and regression tree models allowed the FNIH researchers to test a wide range of muscle variables, identify the best predictors of mobility disability, and propose sex-specific cut-points for discriminating between normal and pathological muscle aging ${ }^{27)}$.

The FNIH initiative recommended defining low muscle mass based on the ratio between appendicular lean mass (ALM) and body mass index or, alternatively, the crude ALM ${ }^{26)}$. Since these definitions might today be considered the current "best practice" for defining low muscle mass in the elderly, the SPRINTT consortium decided to follow the FNIH recommendations. Internal analyses were also conducted of the existing data by the SPRINTT partners with the aim of understanding whether it was best to choose one of the 2 criteria or simultaneously consider both ${ }^{28)}$. In general, the adoption of the only $A_{L} M_{B M I}$ definition might have enriched the SPRINTT target population with obese sarcopenic individuals but might have missed the inclusion of sarcopenic individuals with a normal-to-low body mass index. The skewing of the sarcopenia definition toward a sarcopenic obesity phenotype might have severely limited the amount of future analyses simply because it did not capture the entire spectrum of the sarcopenia manifestation. At the same time, choosing the crude ALM definition alone might have exposed the SPRINTT project to the criticism of not having adhered to the strongest evidence/recommendation. For these reasons, the consortium agreed to determine the skeletal muscle mass reduction whenever the individual presents at least 1 of the 2 criteria.

\section{Physical Impairment or Frailty}

As mentioned above, the concept of physical frailty of interest for the SPRINTT project had to be characterized by a special and direct focus on the skeletal muscle. The need to define the qualitative aspect of sarcopenia with an organ-specific measure led the SPRINTT consortium to discard the traditional definitions of frailty. They were considered too multidimensional to adequately meet the needs of a regulatory agency-acceptable construct. The frailty phenotype (usually considered a marker of physical frailty) did not guarantee a mono-dimensional evaluation of the individual's vul- nerability. In fact, it has been argued that its constituent items may not present equal weight, relevance, and meaning, in particular when interacting with one another ${ }^{29)}$. It is also paradigmatic how the physical sign of fatigue/exhaustion is frequently measured using items derived from scales/questionnaires assessing depressive symptoms. A cognitive pattern is thus implicitly included in this defining criterion, leading it to capture something beyond the physical domain.

In the absence of a "traditional definition" of frailty that exclusively focuses on the physical domain of the individual, the consortium looked elsewhere and found in the short physical performance battery (SPPB) an optimal candidate for this purpose. The SPPB was developed in the 1990s to objectively measure lower-extremity physical performance ${ }^{30}$. Although the SPPB is not traditionally considered a measure of frailty, it still adequately responds to the need of detecting older persons with an enhanced vulnerability to stressors and exposes one to a higher risk of negative health-related events (including disability) ${ }^{30-33)}$. In the absence of a clear "gold standard" assessment tool for frailty, the focus on physical function impairment may represent a valid appro$\mathrm{ach}^{34)}$ since the physical domain is considered to represent the main core feature of frailty ${ }^{35}$. Moreover, the assessment of physical performance has become the standard practice when frailty is measured ${ }^{36)}$, especially when the latter is considered a predisability condition.

By convention, an older person with an SPPB score $>9$ is considered robust ${ }^{34)}$. Conversely, a score $\leq 9$ identifies individuals at increased risk of negative outcomes (i.e., frailty). The lower the score, the worse the individual's physical performance. Consistently, an SPPB score $\leq 7$ is commonly used to define a subgroup of particularly frail individual ${ }^{377}$. In SPRINTT, we proposed the use of an SPPB score of 3-9 to identify physically frail individuals. This choice would have allowed the exclusion of those subjects who were either too robust or too dependent. In fact, it has been demonstrated that an SPPB score $<3$ is generally incompatible with one's ability to complete the $400-\mathrm{m}$ walk test (primary endpoint for the SPRINTT trial; see below) ${ }^{38)}$. The range of physical impairment chosen in SPRINTT allows researchers to capture a gray area of physical performance, the status of the individual who is still able to maintain an adequate level of independence but is at an increased risk of disability ${ }^{38)}$. In the SPRINTT trial, special attention is given to the frailest participants (those with an SPPB score $\leq 7$ ) with an oversampling of these individuals. The available evidence shows that persons with an SPPB score $\leq 7$ may be more likely to express the largest effect size from the intervention ${ }^{37,39)}$.

\section{Absence of Disability}

The primary outcome of the SPRINTT trial will be defined 
by the incidence of mobility disability. This condition is operationalized as the onset of incapacity at completing a $400-\mathrm{m}$ walk test within 15 minutes ${ }^{40-42)}$. Therefore, the ability to pass this test at the study entry automatically defined the third key inclusion criterion in the operationalization of the target condition (i.e., PF\&S).

The choice of the $400-\mathrm{m}$ walk test as a measure of the primary outcome in SPRINTT is motivated by several reasons. The target distance of $400 \mathrm{~m}$ was chosen because it represents the mean distance that a healthy older adult can cover in 6 minutes ${ }^{43}$. The $400-\mathrm{m}$ walk test is a strong predictor of major negative health-related events (including disability and mortality) independent of comorbidities. This test is considered highly discriminative for the functional assessment of even apparently well-functioning older adults ${ }^{40-42)}$. This definition of mobility disability was used in prior geriatric clinical trials of major impact in the field, in particular the LIFE studies $^{37,39)}$. It is also noteworthy that the test is designed to provide a dichotomous image (i.e., capacity/incapacity to complete the task) of the clinically relevant condition of mobility disability ${ }^{40-42)}$. Moreover, a further reason for choosing the $400-\mathrm{m}$ walk test is that mobility disability represents the first clinically relevant step in the disabling cascade, implying greater sensitivity to the benefits coming from interventions. In the 400-m walk test, the individual is asked to complete the task within 15 minutes. In our SPRINTT experience and consistent with the literature ${ }^{44)}$, the person who fails the test usually stops it within the first 5-8 minutes; it is quite unlikely that one will fail the test upon approaching the maximum time limit. In other words, the 15-minute limit is there not to stress the individual and challenge his/her reserves but to put an end to a task that a slow walker will be quite unlikely to complete. This is a reason for which the $400-\mathrm{m}$ walk test was preferred by the SPRINTT consortium over the 6-minute walk test (6MWT). In fact, the two tests are very different in their nature. The 6MWT is designed to stress the cardiorespiratory capacities of the individual to quantify physiological reserves and exercise tolerance. The test measures the capacity of the subject to cope with challenging and prolonged stressors. Differently, in the 400-m walk test, the individual is asked to walk for a predefined distance at his most convenient pace. Therefore, the test does not challenge the physiological reserves of the subject but simply measures his/her capacity to function (i.e., reach the preset and specific goal of a 400-m distance).

\section{CONCLUSION}

The SPRINTT project is currently ongoing. Participant recruitment will end in summer 2017, and the study interventions will be conducted until January 2019. To date, in the absence of available official data, it is only possible to state (based on in-field experience) that the population captured by the definition of PF\&S: (1) exists (as demonstrated by the active participants' recruitment across Europe); (2) presents a clear unmet clinical need (consistently with the observed physical impairment); and (3) is characterized by a strong connotation of muscular decline (due to the presence of sarcopenia per eligibility criteria). This population is still independent in daily life but presents a risk profile that is strongly characterized by muscular decline. The autonomy preserved by these persons paradoxically keeps them out of the healthcare services, so their needs remain largely unmet. Traditional healthcare systems (designed to be reactive against a clear clinical manifestation) do not consider them as in need of preventive or therapeutic intervention. Leaving them alone without answers to their needs simply means passively waiting for the catastrophic manifestation of disability. As repeatedly stated elsewhere ${ }^{5,45)}$, it is time to act by developing novel strategies to counteract the disabling cascade.

To our knowledge, SPRINTT represents a unique research initiative that has led regulatory agencies to discuss with the geriatric world a new model of "disease" focused on physical function loss. It is important that discussions continue with all major stakeholders to reshape the traditional medical approach and give greater value to the functiondriven expertise of geriatricians ${ }^{46)}$.

Conflicts of Interest Disclosures: The researchers claim no conflicts of interest.

\section{REFERENCES}

1. Old and frail. Br Med J 1968;1:723-4.

2. Fried LP, Tangen CM, Walston J, Newman AB, Hirsch C, Gottdiener J, et al. Frailty in older adults: evidence for a phenotype. J Gerontol A Biol Sci Med Sci 2001;56:M146-56.

3. Mitnitski AB, Mogilner AJ, Rockwood K. Accumulation of deficits as a proxy measure of aging. ScientificWorldJournal 2001;1:323-36.

4. Cesari M, Marzetti E, Thiem U, Pérez-Zepeda MU, Abellan Van Kan G, Landi F, et al. The geriatric management of frailty as paradigm of "The end of the disease era". Eur J Intern Med 2016;31:11-4.

5. Morley JE, Vellas B, van Kan GA, Anker SD, Bauer JM, Bernabei R, et al. Frailty consensus: a call to action. J Am Med Dir Assoc 2013;14:392-7.

6. Theou O, Brothers TD, Mitnitski A, Rockwood K. Operationalization of frailty using eight commonly used scales and comparison of their ability to predict all-cause mortality. J Am Geriatr Soc 2013;61:1537-51.

7. Hoogendijk EO, van der Horst HE, Deeg DJ, Frijters DH, Prins BA, Jansen AP, et al. The identification of frail older adults in primary care: comparing the accuracy of five simple instruments. Age Ageing 2013;42:262-5. 
8. Rosenberg IH. Sarcopenia: origins and clinical relevance. J Nutr 1997;127(5 Suppl):990S-991S.

9. Herndon LA, Schmeissner PJ, Dudaronek JM, Brown PA, Listner KM, Sakano Y, et al. Stochastic and genetic factors influence tissue-specific decline in ageing C. elegans. Nature 2002; 419:808-14.

10. Ferrucci L, de Cabo R, Knuth ND, Studenski S. Of Greek heroes, wiggling worms, mighty mice, and old body builders. J Gerontol A Biol Sci Med Sci 2012;67:13-6.

11. Lim JY. Sarcopenia: an emerging giant greater than osteoporosis. Ann Geriatr Med Res 2016;20:167.

12. Anker SD, Morley JE, von Haehling S. Welcome to the ICD10 code for sarcopenia. J Cachexia Sarcopenia Muscle 2016;7: $512-4$.

13. Rolland Y, Dupuy C, Abellan van Kan G, Gillette S, Vellas B. Treatment strategies for sarcopenia and frailty. Med Clin North Am 2011;95:427-38, ix.

14. Cesari M, Landi F, Vellas B, Bernabei R, Marzetti E. Sarcopenia and physical frailty: two sides of the same coin. Front Aging Neurosci 2014;6:192.

15. Bauer JM, Sieber CC. Sarcopenia and frailty: a clinician's controversial point of view. Exp Gerontol 2008;43:674-8.

16. Cesari M, Prince M, Thiyagarajan JA, De Carvalho IA, Bernabei R, Chan P, et al. Frailty: an emerging public health priority. J Am Med Dir Assoc 2016;17:188-92.

17. Cesari M, Vellas B. Sarcopenia: a novel clinical condition or still a matter for research? J Am Med Dir Assoc 2012;13:766-7.

18. Marzetti E, Calvani R, Landi F, Hoogendijk EO, Fougère B, Vellas B, et al. Innovative medicines initiative: The SPRINTT Project. J Frailty Aging 2015;4:207-8.

19. Landi F, Calvani R, Cesari M, Tosato M, Martone AM, Bernabei $\mathrm{R}$, et al. Sarcopenia as the biological substrate of physical frailty. Clin Geriatr Med 2015;31:367-74.

20. Landi F, Cesari M, Calvani R, Cherubini A, Di Bari M, Bejuit $\mathrm{R}$, et al. The "Sarcopenia and Physical fRailty IN older people: multi-componenT Treatment strategies" (SPRINTT) randomized controlled trial: design and methods. Aging Clin Exp Res 2017;29:89-100.

21. Cesari M, Pérez-Zepeda MU, Marzetti E. Frailty and multimorbidity: different ways of thinking about geriatrics. J Am Med Dir Assoc 2017;18:361-4.

22. Cruz-Jentoft AJ, Baeyens JP, Bauer JM, Boirie Y, Cederholm T, Landi F, et al. Sarcopenia: European consensus on definition and diagnosis: Report of the European Working Group on Sarcopenia in Older People. Age Ageing 2010;39:412-23.

23. Fielding RA, Vellas B, Evans WJ, Bhasin S, Morley JE, Newman $\mathrm{AB}$, et al. Sarcopenia: an undiagnosed condition in older adults. Current consensus definition: prevalence, etiology, and consequences. International working group on sarcopenia. J Am Med Dir Assoc 2011;12:249-56.

24. Muscaritoli M, Anker SD, Argilés J, Aversa Z, Bauer JM, Biolo $\mathrm{G}$, et al. Consensus definition of sarcopenia, cachexia and precachexia: joint document elaborated by Special Interest Groups (SIG) "cachexia-anorexia in chronic wasting diseases" and "nutrition in geriatrics". Clin Nutr 2010;29:154-9.

25. Morley JE, Abbatecola AM, Argiles JM, Baracos V, Bauer J, Bhasin S, et al. Sarcopenia with limited mobility: an international consensus. J Am Med Dir Assoc 2011;12:403-9.
26. Studenski SA, Peters KW, Alley DE, Cawthon PM, McLean RR, Harris TB, et al. The FNIH sarcopenia project: rationale, study description, conference recommendations, and final estimates. J Gerontol A Biol Sci Med Sci 2014;69:547-58.

27. Cawthon PM, Peters KW, Shardell MD, McLean RR, Dam TT, Kenny AM, et al. Cutpoints for low appendicular lean mass that identify older adults with clinically significant weakness. J Gerontol A Biol Sci Med Sci 2014;69:567-75.

28. Cesari M, Landi F, Calvani R, Cherubini A, Di Bari M, Kortebein $\mathrm{P}$, et al. Rationale for a preliminary operational definition of physical frailty and sarcopenia in the SPRINTT trial. Aging Clin Exp Res 2017;29:81-8.

29. Hoogendijk EO, van Kan GA, Guyonnet S, Vellas B, Cesari M. Components of the frailty phenotype in relation to the frailty index: results from the toulouse frailty platform. J Am Med Dir Assoc 2015;16:855-9.

30. Guralnik JM, Ferrucci L, Simonsick EM, Salive ME, Wallace RB. Lower-extremity function in persons over the age of 70 years as a predictor of subsequent disability. $\mathrm{N}$ Engl J Med 1995;332:556-61.

31. Guralnik JM, Simonsick EM, Ferrucci L, Glynn RJ, Berkman LF, Blazer DG, et al. A short physical performance battery assessing lower extremity function: association with self-reported disability and prediction of mortality and nursing home admission. J Gerontol 1994;49:M85-94.

32. Guralnik JM, Ferrucci L, Pieper CF, Leveille SG, Markides KS, Ostir GV, et al. Lower extremity function and subsequent disability: consistency across studies, predictive models, and value of gait speed alone compared with the short physical performance battery. J Gerontol A Biol Sci Med Sci 2000;55:M221-31.

33. Cesari M, Kritchevsky SB, Newman AB, Simonsick EM, Harris TB, Penninx BW, et al. Added value of physical performance measures in predicting adverse health-related events: results from the Health, Aging And Body Composition Study. J Am Geriatr Soc 2009;57:251-9.

34. Studenski S, Perera S, Wallace D, Chandler JM, Duncan PW, Rooney E, et al. Physical performance measures in the clinical setting. J Am Geriatr Soc 2003;51:314-22.

35. Ferrucci L, Guralnik JM, Studenski S, Fried LP, Cutler GB Jr, Walston JD, et al. Designing randomized, controlled trials aimed at preventing or delaying functional decline and disability in frail, older persons: a consensus report. J Am Geriatr Soc 2004;52:625-34.

36. Abizanda P, Romero L, Sánchez-Jurado PM, Atienzar-Núñez P, Esquinas-Requena JL, García-Nogueras I. Association between Functional Assessment Instruments and Frailty in Older Adults: The FRADEA Study. J Frailty Aging 2012;1:162-8.

37. Pahor M, Guralnik JM, Ambrosius WT, Blair S, Bonds DE, Church TS, et al. Effect of structured physical activity on prevention of major mobility disability in older adults: the LIFE study randomized clinical trial. JAMA 2014;311:2387-96.

38. Vasunilashorn S, Coppin AK, Patel KV, Lauretani F, Ferrucci L, Bandinelli S, et al. Use of the Short Physical Performance Battery Score to predict loss of ability to walk 400 meters: analysis from the InCHIANTI study. J Gerontol A Biol Sci Med Sci 2009;64:223-9.

39. LIFE Study Investigators, Pahor M, Blair SN, Espeland M, Fielding R, Gill TM, et al. Effects of a physical activity inter- 
vention on measures of physical performance: Results of the lifestyle interventions and independence for Elders Pilot (LIFE-P) study. J Gerontol A Biol Sci Med Sci 2006;61:1157-65.

40. Sayers SP, Guralnik JM, Newman AB, Brach JS, Fielding RA. Concordance and discordance between two measures of lower extremity function: 400 meter self-paced walk and SPPB. Aging Clin Exp Res 2006;18:100-6.

41. Rejeski WJ, Fielding RA, Blair SN, Guralnik JM, Gill TM, Hadley EC, et al. The lifestyle interventions and independence for elders (LIFE) pilot study: design and methods. Contemp Clin Trials 2005;26:141-54.

42. Chang M, Cohen-Mansfield J, Ferrucci L, Leveille S, Volpato $\mathrm{S}$, de Rekeneire $\mathrm{N}$, et al. Incidence of loss of ability to walk 400 meters in a functionally limited older population. J Am
Geriatr Soc 2004;52:2094-8.

43. Simonsick EM, Montgomery PS, Newman AB, Bauer DC, Harris T. Measuring fitness in healthy older adults: the Health $A B C$ Long Distance Corridor Walk. J Am Geriatr Soc 2001;49:1544-8.

44. Rolland YM, Cesari M, Miller ME, Penninx BW, Atkinson HH, Pahor M. Reliability of the 400-m usual-pace walk test as an assessment of mobility limitation in older adults. J Am Geriatr Soc 2004;52:972-6.

45. Lee Y. Evidence-based prevention of frailty in older adults. J Korean Geriatr Soc 2015;19:121-9.

46. Marzetti E, Sanna T, Calvani R, Bernabei R, Landi F, Cesari M. Brand new medicine for an older society. J Am Med Dir Assoc 2016;17:558-9. 